\title{
A BEAMLINE ANALYSIS PROGRAM FOR MAIN INJECTOR COMMISSIONING ${ }^{\dagger}$
}

\author{
M.J. Yang $\ddagger$ FNAL, Batavia, IL
}

\begin{abstract}
From the start of Fermilab Main Injector commissioning in October of 1998 an on-line application program has been used to collect data from the accelerator control system. This is a comprehensive program that collects accelerator data, does modeling calculation, and performs analysis of the optical properties of the beamline. Data and calculation result are viewed with graphic display or text listing. This program is responsive and flexible. Interface is provided for modifying beamline setup, as well as importing from and exporting to MAD program.
\end{abstract}

\section{INTRODUCTION}

The accelerator modeling programs used for Fermilab Main Injector project, such as MAD [1] and TEAPOT [2], do not deal directly with actual data. This write-up describes an ACNET [3] console application program that fully integrated beamline setup, data acquisition, data storage, calculation, analysis, and display. It is responsive, requires minimum human intervention, and has sufficient flexibility. Extensive graphic displays are provided for viewing data and calculated result. Text listing is an alternative which also provides the means to e-mail data to user.

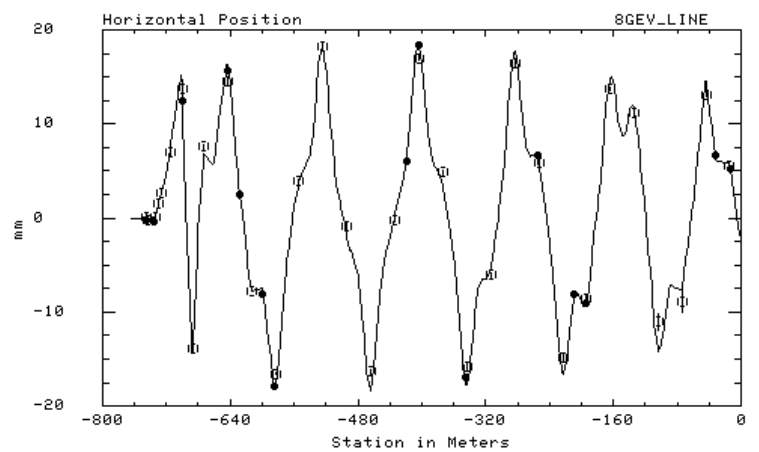

Figure 1. The horizontal plane orbit in the Main Injector $8-\mathrm{GeV}$ transfer line. Circles are data from BPM and solid dots from the profile monitors. The connected line is the calculated orbit.

During the Main Injector commissioning this program has successfully demonstrated its ability to handle data and to provide calculation that reproduces data well [4] [5]. Figure 1 is an example of orbit data and calculation taken from the MI 8-GeV transfer line. Figure 2 shows one-bump data taken from the Main Injector ring at 8$\mathrm{GeV}$ energy. Other data examples will be shown as each components of the program is described.

\section{BEAM LINE SETUP}

The program provides user the ability to edit devices and to configure beamline setup. New setup can be imported

\footnotetext{
$\dagger$ Work supported by the US Department of Energy under contract DE-AC02-76CH00300.

$\ddagger$ E-mail: YANG@FNAL.GOV
}

from MAD program files. Existing setup can be exported in MAD input file format for calculation.

Beamline entities such as Beamline, Segment, Element, and Devices are used in the program. Figure 3 illustrates the relationships between these entities.

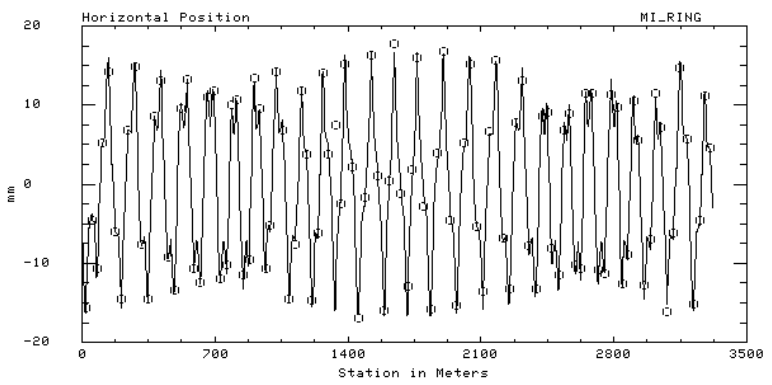

Figure 2. One-bump orbit data taken from the Main Injector. The circles are the BPM data and the connected line is the closed orbit calculation based on the design lattice.

\subsection{Beamline devices}

Two types of devices are used in the program. Physical devices are used in the actual beamline setup. Supplemental devices supply information for the physical devices to implement its functionality.

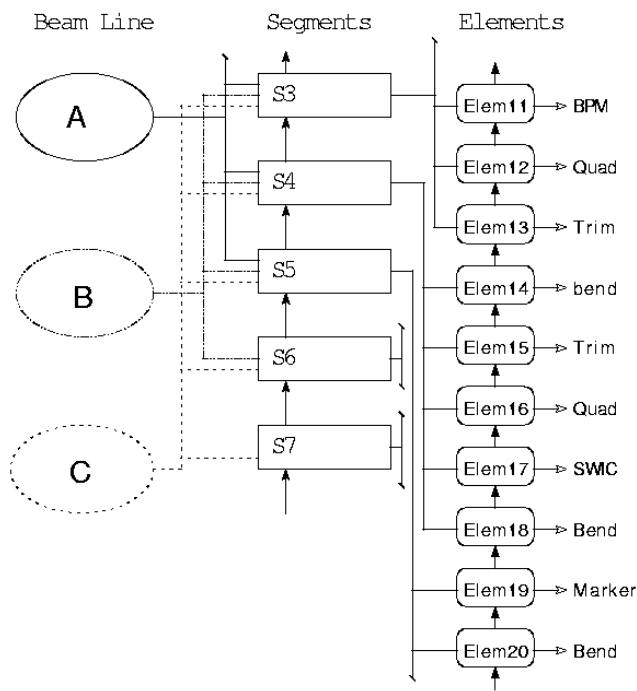

Figure 3. Example of beamline setup hierarchy used in the analysis program for calculation, display, and text listing. Beamline "B" includes exactly segments S3, S4, S5, and S6. "C" is all that plus S7. "A" has segments preceding S3 as well.

Physical devices are subdivided into three categories. Passive devices such as BPMs and Profile monitors keep beam data but do nothing to the beam. Active devices are dipoles, quadrupoles, lambertsons, and electrostatic septa. Others include Aperture device that is active only for phase space tracking and Marker that does nothing.

There are three kinds of supplemental devices. Database devices are used for data acquisition. Through refer- 
ence to the database device the beamline device gets its information. Changes to database devices are reflected by the calculation automatically. Multipole device provides integrated strengths in dipole, quadrupole, sextupole, and octupole field for active beamline devices. Bump device modifies database value to be referenced by other devices.

\subsection{Elements}

Element is the basic block in the calculation and is where data and calculation result are stored. Its function depends on its association to beamline device. Elements are sequentially ordered such that each points to its predecessor. An element belongs only to one segment.

\subsection{Segments}

Segments are also sequentially ordered. Each points to its own predecessor. It can be in multiple beamlines but only once in any one beamline. A valid segment contains a number of consecutive elements.

\subsection{Beamlines}

This is the entity on which all calculation, display, and listing are based. A valid beamline includes a number of consecutive segments.

\section{DATA ACQUISITION}

BPM and profile monitor data are needed for optics analysis. Magnet settings and readings are needed for lattice calculation. Other information such as intensity monitor data is also acquired to further document the state of the machine. All relevant data is acquired through the Fermilab accelerator control system with efficiency.

Multiple samples of the beamline at different states during a study of machine responses are supported. The data is analyzed on-line or saved for off-line analysis.

\section{CALCULATION}

Calculations are done for both the upstream and downstream of each beamline element, whenever database devices are modified. Fast response and minimum user intervention are important considerations in the program.

\subsection{MAD compatibility}

The calculation result of the program has been verified to be identical to that of the MAD program except in two cases. The first, MAD program retains the residual roll in its reference coordinate frame after skew dipole and that affects all down-stream elements. Secondly, appreciable difference exists in the calculation of twiss parameters when a skew quadrupole is included in the beamline.

\subsection{Live}

The program is designed such that the lattice calculation is updated using the acquired magnet current data. The recalculation starts the instant new data is loaded.

\subsection{Lattice}

Beam particle transport is the means of all calculation in this program. Execution starts with a chosen beamline, its segments, and to the elements. Element invokes the asso- ciated device to do the actual transport. Twiss parameters, for example, are calculated by tracking four simultaneous beam particles of different initial conditions.

The program calculates transfer line orbit, closed orbit for synchrotron, twiss parameters, dispersion function, and others. The difference between calculation and the available data is updated along with the RMS deviation.

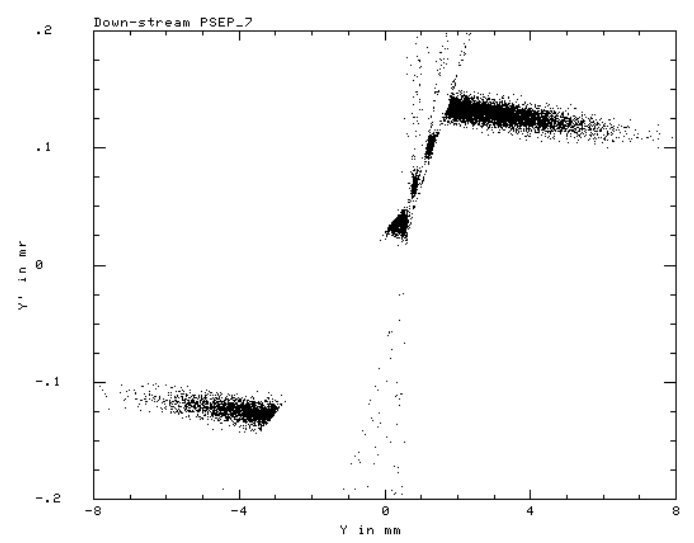

Figure 4. Vertical phase-space plot of result from tracking 10,000 beam particles in a 3-way split simulation.

\subsection{Tracking}

Phase space tracking simulates non-analytical effects due to devices such as electro-static beam splitting septum or aperture restrictions. Figure 4 shows the result of tracking 3-way split through seven septa, as are used in Fermilab Fixed-Target beamline. In this case the three downstream septa used for the second split were poorly aligned and the scattering of beam particles is evident. Figure 5 shows the would-be signal from a profile monitor.

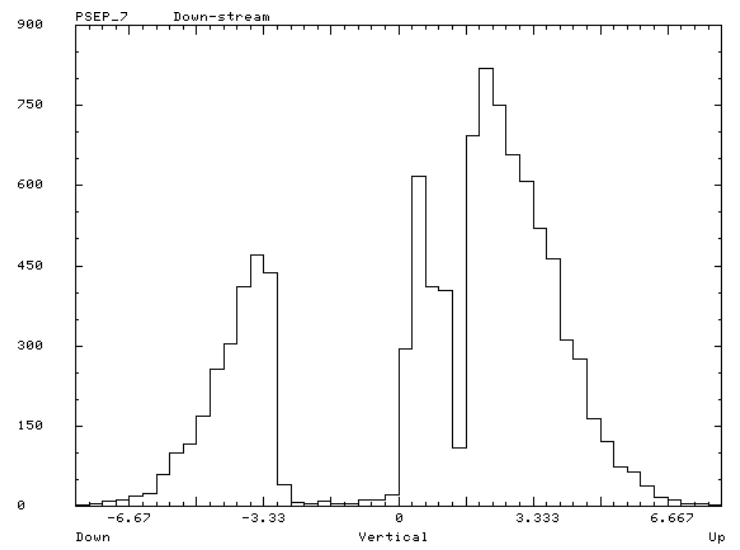

Figure 5. Histogram display here simulates the profile monitor response with tracking result as shown in Figure 4.

\subsection{Site layout}

Site layout calculates the invisible reference trajectory that is assumed in the beam particle transport. Only the dipole bend angles are used to calculate the 3-dimensional site coordinate for each beamline element.

\section{ANALYSIS}

The program provides user the ability to scan through data sets efficiently to identify potentially flawed data set. Analyses supported in the program are described here. 


\subsection{Quadrupole diagnostics}

Quadrupole strengths affect the beta function and can be verified using the one-bump orbit data. Consistency between data and calculation is a necessary condition. If available data uniquely determines the quadrupole strengths a sufficient condition is also established. The consequence of having only necessary condition in the lattice analysis will be mentioned in Section 5.4.
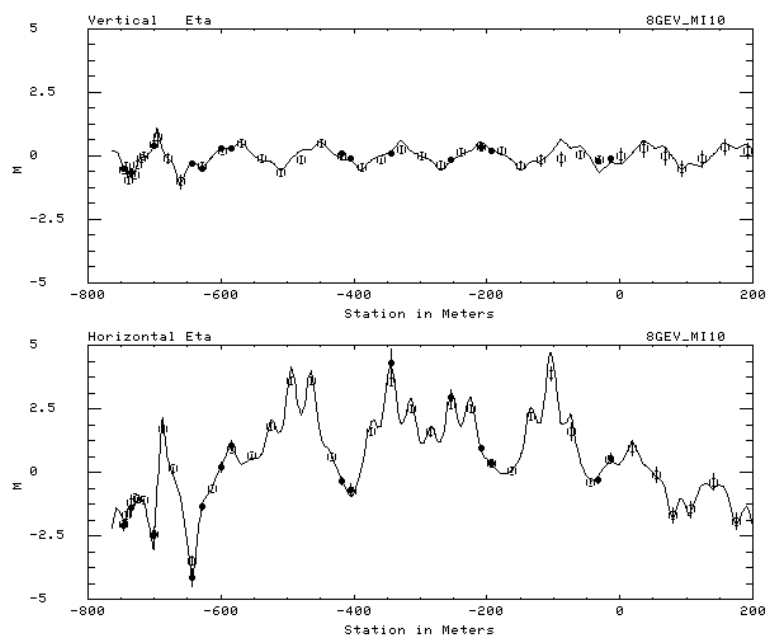

Figure 6. The dispersion function measurement data and the calculation using the optimized initial condition.

\subsection{Dispersion function}

The measurement of dispersion function requires only the knowledge of momentum error used and its accuracy. Figure 5 shows the dispersion function as measured from the new Main Injector 8-GeV transfer line. In a transfer line the initial condition, the bends, and the lattice all contribute to modifying the dispersion function.

\subsection{Phase advance and beta function}

This analysis applies only for the circular machine. In the first step, the BPM data is analyzed against the kick angle of the corrector to get the kick coefficient in " $\mathrm{mm} / \mathrm{mr}$ ". To do the phase analysis at least two data sets from different correctors are needed. Beta function can be calculated using only one data set. The phase advance used can be from data or from model calculation.

\subsection{Emittance and lattice function}

Beam profile width is used for the analysis of transfer line emittances. Given a set of initial twiss parameters and initial dispersion functions the program fits for the emittance and $\sigma_{\mathrm{p}} / \mathrm{p}$. Figure 7 plots the beam width sigma data from the $8-\mathrm{GeV}$ transfer line and the calculation using fitted emittances and the optimized initial conditions.

This analysis has important implication on the projected lattice function at the end of the transfer line. As mentioned in Section 5.1, the quadrupoles affects the beta function at monitor locations and therefore the outcome. When the quadruple strengths is not uniquely determined it is still possible that the fitted emittance is free of error. The optimized initial lattice parameters or the projected lattice function, on the other hand, could be in doubt depending on the location of quadrupole uncertainty.

\subsection{Miscellaneous}

The program offers other analyses that may also be useful. Comparing orbit data against calculation can provide information for BPM calibration. Kick impact coefficient and data correlation can also be calculated.
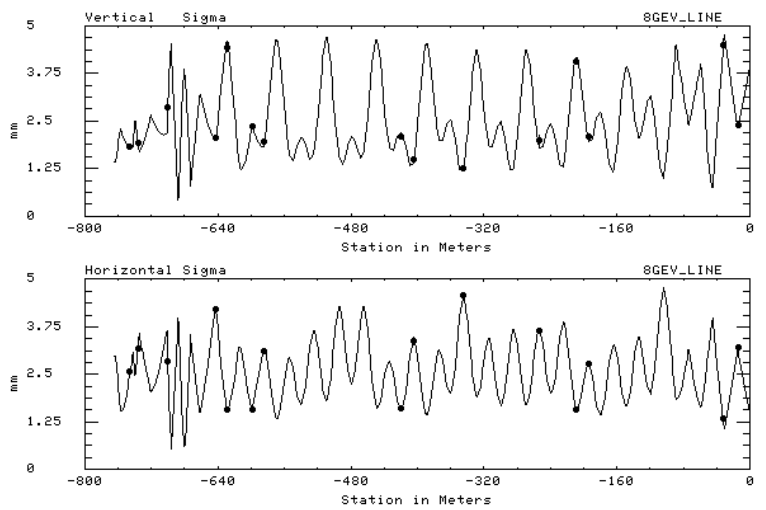

Figure 7. Result of fitting beam emittance to the sigma from profile data from the MI $8-\mathrm{GeV}$ line, using the lattice which best matched the orbit data.

\section{SUMMARY}

The beamline analysis program as described above has performed well as a tool to study transverse beam optics. It is a stand along program with EXCEL like response. It runs on the VAX/VMS, the platform that runs the Fermilab accelerator control system. Its code is optimized to have flexible linkage and yet quick turn-around time for calculation. Features like individual magnet error handling already exit but yet to be explored.

Its ability to import from MAD program output is handy for starting a new setup that is identical to a known calculation. Its internal linkage, however, is vastly different and in order to achieve all the on-line capabilities further setup is required. The program is designed to work on most of the beamlines around Fermilab, with proper setup effort. The flexibility and many display features also make it well suited as an initial designing tool.

\section{ACKNOWLEGEMENTS}

The author would like to thank G. Goderre for his valuable advises on lattice calculation and analysis.

\section{REFERENCES}

[1] Hans Groted and F.C. Iselin. The MAD Program, 1990, CERN SL/90-13 (AP).

[2] L. Schachinger and R. Talman, Particle Accelerator 22, 1987.

[3] Accelerator Control NETwork, FNAL.

[4] S. Assadi, F. Tecker, M.J. Yang, "Optics Measurement of the FNAL Main Injector 8-GeV Transfer Line”, Particle Accelerator conference, 1999.

[5] S. Assadi, C.S. Mishra, F. Tecker, M.J. Yang, "Lattice Function Main Injector at 8-GeV”, Particle Accelerator conference, 1999. 